\title{
Transmission of Schistosoma mansoni in Tikur Wuha area, Southern Ethiopia
}

\author{
Habtamu Mitiku' ${ }^{1}$, Mengistu Legesse ${ }^{2}$, Zelalem Teklemariam ${ }^{1}$, Berhanu Erko ${ }^{2}$
}

\begin{abstract}
Background: Although the epidemiology of schistosomiasis is well established and the disease distribution has also been mapped in Ethiopia, discovery of new foci has continuously been reported.

Objectives: The objective of this study was to assess the establishment of transmission of schistosomiasis mansoni in Tikur Wuha area, southern Ethiopia.

Methods: A cross-sectional epidemiological study involving 375 school children in Tikur Wuha Elementary School was conducted in December 2007 and January 2008. Stool specimens were collected and microscopically examined using Kato-Katz method. Snail survey was also conducted using scoop in Tikur Wuha River and littoral zone of Lake Awassa on the side of Tikur Wuha Kebele (administrative unit). The snails collected were checked for trematode infection by shedding. Laboratory-bred mice were exposed to schistosome cercariae and definite identification of the schistosome was made using eggs and adult worm morphology.

Results: The prevalence and intensity of schistosomiasis mansoni was 12\% and 69 eggs per gram (epg) of stool, respectively. Biomphalaria sudanica collected in Tikur Wuha River shed schistosome cercariae. Adult S. mansoni worms were harvested from laboratory-bred mice after 6 weeks of laboratory maintenance.

Conclusion: The prevalence and intensity of schistosomiasis mansoni among school children was low and the area represents low-risk community. The finding of $S$. mansoni infected young children, the collection of $B$. sudanica infected with schistosome cercariae, and the establishment of infection in lab-bred mice all confirmed the transmission of schistosomiasis mansoni in Tikur Wuha area. Appropriate intervention measures need to be in place to reduce morbidity and transmission of intestinal schistosomiasis in the area. [Ethiop. J. Health Dev. 2010;24(3):180-184]
\end{abstract}

\section{Introduction}

An estimated 207 million cases of schistosomiasis occur in 74 countries of the world, of which about $90 \%$ live in sub-Saharan Africa where Schistosoma haematobium and S. mansoni are endemic (1). In Africa, the mortality attributable to urinary schistosomiasis are estimated to be 150,000 per year, and the number of people dying as a result of intestinal schistosomiasis all estimated to be 130,000 per year (2). Although mortality due to schistosomiasis is low compared to millions of cases, the disease has considerable impact on public health and socio-economic development (3).

In Ethiopia, intestinal schistosomiasis caused by $S$. mansoni and urinary schistosomiasis caused by $S$. haematobium have been known to be endemic. The former is widely distributed in the country while the latter has so far been reported only from three lowland areas, including the Awash and Wabe Shebele valleys and an intermittently flowing stream in Kurmuk at the Ethio-Sudan border $(4-12)$. The main determinants for the distribution, transmission and spreading of the schistosomiasis in Ethiopia include water temperature, local absence or presence of snail intermediate host, human population movement and water impoundment for irrigation and power $(10,11,13,14)$.

A number of schistosomiasis and snail surveys have been carried out in different water bodies in Ethiopia (4-11,
13-16). These previous studies have shown the occurrence of infected subjects and snail intermediate hosts as well as the establishment of intestinal schistosomiasis transmission in different localities of the country.

Although Schistosoma mansoni infection was reported in children younger than 15 years in Lake Awassa and Tikur Wuha areas $(5,9)$ and the occurrence of Biomphalaria sudanica in Tikur Wuha river was also known $(10,11)$, the establishment of schistosomiasis mansoni transmission in the area has not been reported. The present study was, therefore, undertaken to assess the transmission of intestinal schistosomiasis in Tikur Wuha area.

\section{Methods \\ Study area and population}

A cross-sectional study was conducted among schoolchildren enrolled at Tikur Wuha Elementary School, south Ethiopia, in December 2007 and January 2008. The school is found in Tikur Wuha Kebele, Shashemene District, some $266 \mathrm{~km}$ to the south of Addis Ababa (Figure 1). The area has an altitude of 1708 meters above sea level (m.a.s.1.). According to local population census conducted in 2007 by Shashemene District Health Office, there were 1830 households, with about a total of 9151 inhabitants in Tikur Wuha Kebele. The inhabitants of the Kebele earn their living as government employees,

\footnotetext{
${ }^{\mathrm{T}}$ Department of Medical Laboratory Technology, Haromaya University, Haromaya, Ethiopia;

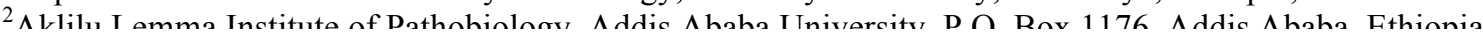


merchants, daily laborers and farmers. Tikur Wuha River and Lake Awassa serve as sources of water for laundering, bathing and other domestic and recreational purposes. The Kebele has only one health post and one elementary school. The total population of the school children attending class during the survey period was 987.

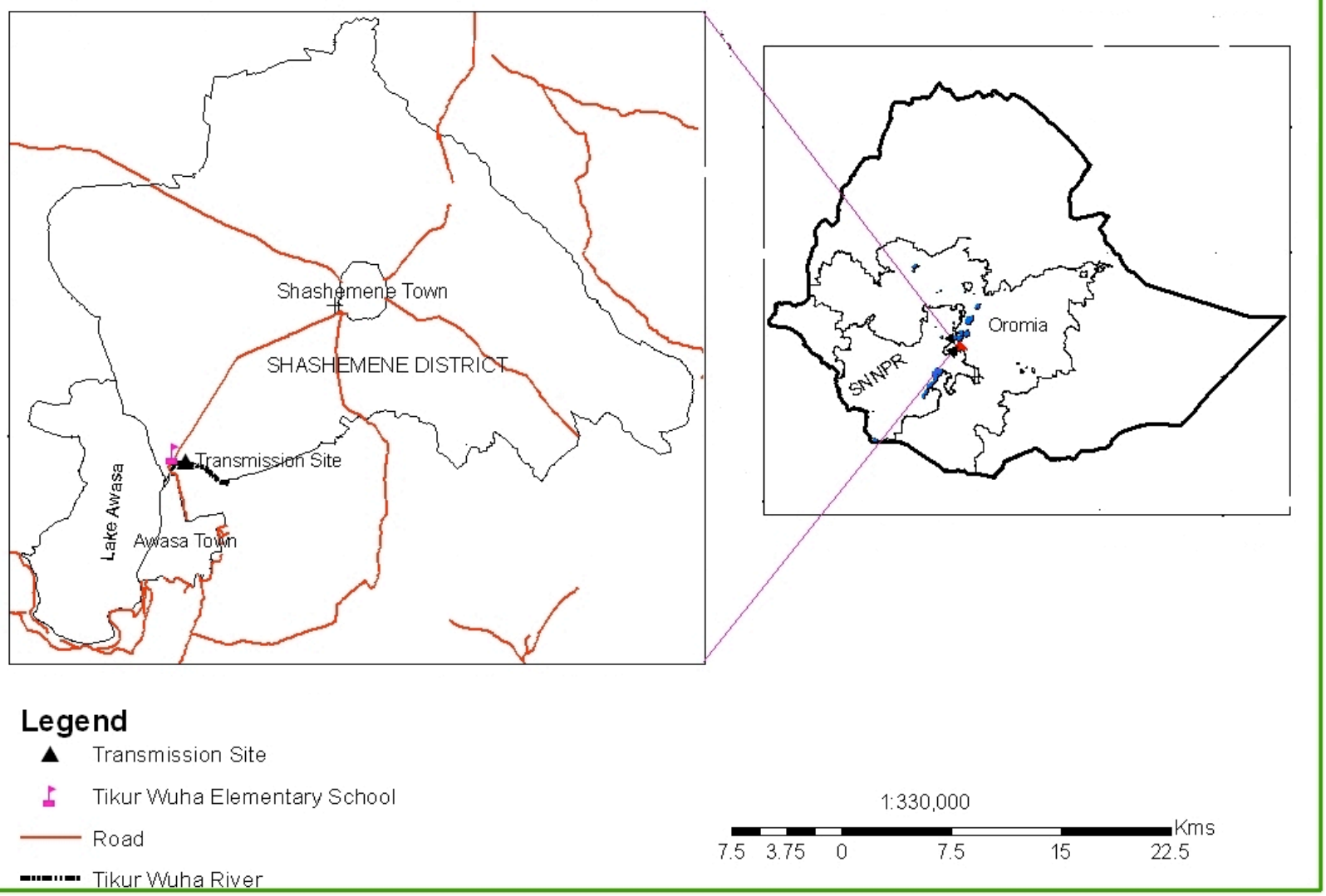

Figure 1. Map of the study site

\section{Sample size and sampling techniques}

The sample size was calculated using the formula $n=Z^{2}$ $\mathrm{P}(1-\mathrm{P}) / \mathrm{d}^{2}$, where $\mathrm{n}$ is sample size, $\mathrm{Z}$ is $95 \%$ confidence interval (1.96), $\mathrm{P}$ is expected prevalence $(50 \%)$ and $\mathrm{d}$ is precision or margin of error (5\%) (17). Since there was no schistosomiasis prevalence report for the area, a prevalence of $50 \%$ was assumed. Hence, the required sample size was computed to be 384 . Nevertheless, 375 (98\%) children participated in the study because 9 selected children refused to do so. The study participants were then selected by systematic random sampling using school roll as a sampling frame.

\section{Stool collection and examination}

Freshly passed stool specimens were collected using clean plastic sheet at the school. Data on age and gender were gathered during stool collection. The principal investigator took part in all processes of stool collection and examination. The stool samples were processed for microscopic examination using Kato-Katz method (template delivering $41.7 \mathrm{mg}$ of stool) (18). Quantitative microscopic examination was done for ova of $S$. mansoni within one week of collection at the Aklilu Lemma Institute of Pathobiology (ALIPB). Egg count per slide was multiplied by 24 to convert into number of eggs per gram (epg) of stool. The intensity of infection was classified according to classes of intensity recommended by WHO (19).

\section{Snail survey}

Snails were surveyed using scoop in Tikur Wuha River and littoral zone of Lake Awassa on the side of Tikur Wuha Kebele. The collected snails were transported to lab at Aklilu Lemma Institute of Pathobiology and identified as Biomphalaria sudanica based on shell morphology. Snails were then checked for trematode infection by exposing them individually to electric light for about 2 hours. In case of emerging cercariae, identification was made to the genus level using tail morphology (20).

\section{Mouse exposure}

Laboratory-bred mice were exposed for 30 minutes to the cercariae shed from the snails. The mice were sacrificed after six weeks of laboratory maintenance and the worms were collected manually from blood vessels around the mesentery. Definite identification of the schistosome 
parasite was made based on the egg and adult worm morphology.

\section{Data analysis}

Data were entered into Microsoft Excel data sheet, checked, edited and cleaned. Then it was exported to STATA version 8.1 for analysis. The Chi-square test was used to test association between prevalence of infection with age and gender while unpaired student's t-test and ftest were used to compare geometric mean egg counts by gender and age groups, respectively.

\section{Ethical considerations}

Ethical clearance for the study was obtained from the Ethical Clearance Committee of Aklilu Lemma Institute of Pathobiology as well as from Shashemene District Health Office, Oromia National Regional State. The objectives of the study were explained to the director, teachers and study participants after which informed consent (assent in case of children younger than 18) was obtained. Only voluntary students were involved in the study. Those children found positive for $S$. mansoni were treated with a single dose of praziquantel $(40 \mathrm{mg} / \mathrm{kg}$ body weight) and those found positive for soiltransmitted helminthiasis were treated with mebendazole.

\section{Results}

A total of 375 children (184 males and 191 females) participated in the study. The age group of the children was 5-17 years, mean age being 10.3 years. Of the total 375 children examined, 45 (12\%) were found positive for $S$. mansoni infection. The highest infection rate $(30.4 \%)$ was observed in children in the 15-17 age group followed by $14.4 \%$ and $4 \%$ for age groups $10-14$ and 5-9 years, in that order. Significant difference was observed between $S$. mansoni infection and age groups $(\mathrm{P}<0.001)$. The prevalence of infection among males and females was $13.6 \%$ and $10.5 \%$, respectively $(\mathrm{P}=0.353)$.

The intensity of $S$. mansoni infection was $69 \mathrm{epg}$. The highest epg was observed among males of 15-17 years while the lowest epg was observed among females of the same age group. Out of 45 children who were positive for S. mansoni infection, $66.7 \%$ had light infection, $31.1 \%$ moderate and $2.2 \%$ heavy intensity of infection. Intensity of $S$. mansoni infection did not differ significantly by gender and age $(\mathrm{P}>0.05)$.

Table 1: Prevalence and intensity of schistosomiasis mansoni by age and gender among children from Tikur Wuha Elementary School, December 20007- January 2008

\begin{tabular}{lcclllllll}
\hline Age Group & \multicolumn{3}{c}{ No. Examined } & \multicolumn{3}{c}{ No. Positive (\%) } & \multicolumn{3}{c}{ epg } \\
\cline { 2 - 10 } Years) & $\mathbf{M}$ & $\mathbf{F}$ & Total & $\mathbf{M}$ & $\mathbf{F}$ & Total & $\mathbf{M}$ & $\mathbf{F}$ & Total \\
\hline $5-9$ & 53 & 70 & 123 & $2(3.8)$ & $3(4.3)$ & $5(4)$ & 76 & 66 & 70 \\
$10-14$ & 117 & 112 & 229 & $20(17)$ & $13(11.6)$ & $33(14.4)$ & 70 & 67 & 69 \\
$15-17$ & 14 & 9 & 23 & $3(21.4)$ & $4(44.4)$ & $7(30.4)$ & 253 & 29 & 73 \\
\hline Total & $\mathbf{1 8 4}$ & $\mathbf{1 9 1}$ & $\mathbf{3 7 5}$ & $\mathbf{2 5 ( 1 3 . 6 )}$ & $\mathbf{2 0 ( 1 0 . 5 )}$ & $\mathbf{4 5 ( 1 2 )}$ & $\mathbf{8 2}$ & $\mathbf{5 6}$ & $\mathbf{6 9}$ \\
\hline
\end{tabular}

The majority of the school children lived in Tikur Wuha Kebele while few came from surrounding peasant associations. Out of 45 students who were infected with S. mansoni, 22\% lived outside Tikur Wuha Kebele while $77.8 \%$ were born and brought up there.

Other intestinal helminth infections which were observed during stool examination included, Trichuris trichiura (32.8\%), Ascaris lumbricoides (29.9), and Enterobius vermicularis $(1 \%)$. Since the slides were not examined within 30 minutes of stool collection, the status of hookworm infections was not known.

Snail survey showed the presence of two snail species, Biomphalaria sudanica and Lymnaea species in Tikur Wuha River. Out of 76 B. sudanica collected from Tikur Wuha, only one shed schistosome cercariae, giving an infection rate of $1.3 \%$. S. mansoni infection was successfully established in laboratory mice and adult worms were harvested after six weeks of laboratory maintenance. No B. sudanica was found along the shore of Lake Awassa during the survey where many people were engaged in laundering, swimming, bathing and fishing activities.

\section{Discussion}

In the present study, the observation that children excreted Schistosoma mansoni ova, the shedding of schistosome cercariae by Biomphalaria sudanica, and the establishment of $S$. mansoni life cycle in laboratory-bred mice all confirmed the establishment of schistosomiasis mansoni transmission in Tikur Wuha River. This focus might have existed for undetermined period of time or a newly established one as a result of ecological changes and population movements.

It has been suggested that population movements from schistosomiasis endemic to non-endemic foci that are ecologically and malacologically receptive areas lead to the establishment of new schistosomiasis focus (14). If Tikur Wuha schistosomiasis focus is a newly established one, this raise the apprehension that schistosomiasis might have spread to a number of new foci previously free of it as also recently reported from several foci (14, $16,21,22)$. 
The prevalence $(12 \%)$ and intensity of schistosomiasis mansoni (69 epg) observed in the present study was lower compared to previous report among children engaged in fishing activities in adjacent Lake Awassa area (9). This difference might be due to differences in occupation and the study subjects. Occupational difference is often reflected in schistosomiasis prevalence as occupational groups performing intensive contact with cercariae-infested water bodies have higher rates of infection (5). Difference in the prevalence of infection in different population groups or segments is also determined by variations in immune levels of individuals. Typically, rates and intensity of infection increased from an early age to a peak around the age 10- 19 years and decrease again in adults $(5,23)$. Nevertheless, immune response might not explain lower prevalence in the present study and among children in Lake Awassa area as the age groups for the two study sites were more or less the same.

There was a general tendency for $S$. mansoni infection rate to increase with age. The highest prevalence was observed among the 15-17 age group, which is in agreement with other studies made in different parts of Ethiopia $(9,16,24-26)$. Such rise in infection with age may correspond with intense human water contact activities.

Previous community studies have shown that males are the most affected groups by schistosomiasis than females $(7,8,24,25)$. This could be explained by the fact that males have higher frequency of contact with cercariae infested water bodies than females. On the other hand, the present study showed that there was no significant difference in prevalence of schistosomiasis between male and female children. This was probably due to similarities in frequency and water contact activities of male and female children in the study area.

Snail surveys showed that Biomphalaria sudanica, intermediate snail hosts of S. mansoni, were abundant in Tikur Wuha River, confirming previous report by Birrie et al. (4). The River was also covered with aquatic weeds, which favor the survival and breeding of the snail hosts. Furthermore, open air defection in vegetation on the river bank was also very common, ensuring completion of the parasite life cycle.

In conclusion, the study has shown transmission of intestinal schistosomiasis in Tikur Wuha River. The prevalence and intensity of schistosomiasis mansoni among school children was low and the area represents low-risk community. Human contaminative activities such as open air defecation and exposure activities such as laundering and bathing favor transmission of schistosomiasis in Tikur Wuha area. Appropriate intervention measures need to be in place to reduce morbidity and transmission of intestinal schistosomiasis in the area.

\section{Acknowledgements}

The study received financial support from Aklilu Lemma Institute of Pathobiology, Addis Ababa University. We would like to thank school director and teachers of Tikur Wuha Elementary School for their cooperation during data collection, and students of Tkur Wuha Elementary School who voluntarily participated in the study. We also gratefully acknowledge Mr Sisay Dessie of the Aklilu Lemma Institute of Pathobiology for his technical assistance and technical staff of Awassa Regional Laboratory for their cooperation during examination of the stool specimens.

\section{References}

1. Steinmann P, Keiser J, Bos R, Tanner M, Utzinger J. Schistosomiasis and water resources development: Systematic review, meta-analysis, and estimates of people at risk. Lancet Infect Dis 2006;6(7):411-425.

2. Fenwick A, Savioli L, Engels D, Bergquist N. R, Todd M. H. Drugs for the Control of Parasitic Diseases: Current Status and Development in Schistosomiasis. Trends. Parasitol 2003;19:509-15.

3. World Health Organization. Preventive chemotherapy in human helminthiasis: coordinated use of anthelminthic drugs in control interventions: A manual for health professionals and programme managers. Geneva: WHO; 2006.

4. Birrie H, Tedla S, Erko B, Berhe N, Abebe F. Schistosomiasis in Finchaa river valley, Wellega region, West Ethiopia. Ethiop $J$ Health Dev 1993;7:9-15.

5. Birrie H, Tedla S, Tilahun G, Kloss H, Eshete H. Schistosomiasis and its distribution in Ethiopia and Eritrea In: Birrie H, Tedla S, Jemaneh L, (Eds.) Schistosomiasis in Ethiopia and Eritrea. Addis Ababa University. 1998;29-89.

6. Ali A, Erko B, Wolde Micheal T, Kloos H. Schistosomiasis In: Berhane Y, Haile Mariam D, Kloos H, (Eds.) Epidemiology and ecology of health and disease in Ethiopia. Ethiopia: Shama Book. 2006;661-673.

7. Roma B, Worku S. Magnitude of Schistosoma mansoni and intestinal helminthic infections among school children in Wondo Genet Zuria, Southern Ethiopia. Ethiop J Health Dev 1997;11:125-129.

8. Wolde Michael T, Endeshaw T, Shibre T, Gebre T, Haddis M, Tilahun D, Gebreyesus L, Dereje S. Intestinal parasitic infection in Western Abaya with special reference with schistosomiasis mansoni. Ethiop J Health Dev 1999;13:21-26.

9. Merid Y, Hegazy M, Mekete G, Tekle Mariam S. Intestinal helmintic infection among children at Lake Awassa Area, south Ethiopia. Ethiop J Health Dev 2001;15:31-37.

10. Birrie H, Lo CT, Erko B, Redda A, Gemeda N. Further investigations on freshwater snails of Ethiopia. SINET: Ethiop J Sci1995;18:195-206.

11. Kloss H, Lo CT, Birrie H, Ayele T, Tedla S, Tseegay F. Schistosomiasis in Ethiopia. Soc Sci Med 1988;26:803-827. 
12. Ali A, Lo CT, Ayele T. Schistosoma haematobium in western Ethiopia. Ethiop Med J. 1986;24:73-78.

13. Alemayehu T, Yeebiyo Y, Ghebreysus T.A, Witten K.H. Malaria, Schistosomiasis and intestinal helminthes in relation to micro dams in Tigray Northern Ethiopia. Parasitology 1998;40:259-267.

14. Erko B, Gemetchu T, Gemeda N, Dessie S. Transmission of intestinal schistosomiasis in Addis Ababa. E Afr Med J 1996; 73:732-734.

15. Erko B, Medhin G, Berhe N, Abebe F, GeberMichael T, Gundersen S.G. Epidemiological studies on Schistosomiasis in Wendo Genet, South Ethiopia. Ethiop Med J 2002; 40: 29-39.

16. Woldemichael $T$, Kebede A. Newly identified endemic areas of schistosomiasis mansoni in Tigray, North Ethiopia. Ethiop Med J 1996; 34: 73-76.

17. Daniel WW. Biostatics a foundation for analysis in the health science. $6^{\text {th }}$ ed. John Willey and Sons Inc., New York, USA; 1995.

18. WHO. Basic Laboratory Methods in Medical Parasitology. World Health Organization, Geneva: WHO; 1991.

19. WHO. Prevention and control of schistosomiasis and soil-Transmitted Helminthiasis. WHO Tech Rep Ser. 2002; 912: 1-57.
20. Frandsen F, Christensen N.O. An introductory guide to the identification of cercariae from African fresh water snails with special reference to cercariae of trematodes species of medical and veterinary importance. Acta Tropica 1984; 41: 118-202.

21. Wodimagegnehu $\mathrm{T}$, Birrie $\mathrm{H}$, Yeneneh $\mathrm{H}$. Schistosomiasis and intestinal helmintic infections in Delo Awraja, Bale administrative region south Ethiopia. Ethiop J Health Dev 1997; 11: 183-188.

22. Erko B, Gebre-michael T, Balcha F, Gundersen SG. Implication of Papio anubis in the Transmission of Intestinal Schistosomiasis in Three New Foci in Kime Area, Ethiopia. Parasitol Int 2001;50(4):259-266.

23. Gryseels B, Polman K, Clerinx J, Kesten L. Human Schistosomiasis. Lancet 2006;368:1106-1118.

24. Erko B, Tedla S, Petros B. Transmission of intestinal schistosomiasis in Bahir Dar, North East Ethiopia. Ethiop Med J 1991;29:199-211.

25. Assefa T, Woldemmicale $T$, Dejene A. Intestinal parasitism among students in three localities in South Wello, Ethiopia. Ethiop $J$ Health Dev 1998;12:231-235.

26. Gundersen SG, Birrie H, Torvite HP, Secherbaum H. Control of schistosomiasis mansoni in the Blue Nile valley of western Ethiopia by mass chemotherapy and focal snail control: a primary health care experience. Trans R Soc Trop Med Hyg 1990; 84: 819-825. 